\title{
COVID-19 pandemic revisited: lessons the radiology community has learned a year later
}

\author{
Soheil Kooraki ${ }^{1} \cdot$ Melina Hosseiny ${ }^{2} \cdot$ Erik M. Velez $^{3} \cdot$ Amit Gupta $^{4} \cdot$ Robert Gilkeson $^{4} \cdot$ Steven S. Raman ${ }^{2}$. \\ Ali Gholamrezanezhad ${ }^{5}$
}

Received: 18 May 2021 / Accepted: 3 September 2021 / Published online: 28 September 2021

(c) American Society of Emergency Radiology 2021

\begin{abstract}
For more than 1 year, COVID-19 pandemic has impacted every aspect of our lives. This paper reviews the major challenges that the radiology community faced over the past year and the impact the pandemic had on the radiology practice, radiologistin-training education, and radiology research. The lessons learned from COVID-19 pandemic can help the radiology community to be prepared for future outbreaks and new pandemics, preserve good habits, enhance cancer screening programs, and adapt to the changes in radiology education and scientific meetings.
\end{abstract}

Keywords COVID-19 $\cdot$ Pandemic $\cdot$ Lessons $\cdot$ Radiology $\cdot$ Resident $\cdot$ Education $\cdot$ Healthcare $\cdot$ Research

\section{Introduction}

The Coronavirus Disease 2019 (COVID-19) pandemic has broadly and deeply impacted every aspect of medical care requiring operational restructuring, resource allocation, and swift adoption of novel technologies and workflows for healthcare systems around the globe. The pandemic has caused an unprecedented strain on healthcare workers (HCW), who have been dealing with vast and ever-changing scientific evidence around SARS-CoV-2, including epidemiology, infectivity, clinical syndromes, and imaging guidelines. COVID-19 was officially declared as a pandemic by the World Health Organization (WHO) on March 11, 2020
[1], when WHO experts called for urgent global action in the following key areas: (1) to prepare and to be ready, (2) to detect, protect and treat, (3) to reduce transmission, and (4) to innovate and learn.

Radiologists have played a key role in the diagnosis of COVID-19 patients and management of complications. However, radiology departments concurrently have faced challenges maximizing departmental safety, while safely maintaining necessary diagnostic and interventional services for patients minimizing risk of departmental SARS-CoV-2 transmission [2, 3]. In addition, many radiology departments had substantially decreased volumes of outpatient diagnostic imaging studies at the beginning of the pandemic in the

1 University of California, Los Angeles (UCLA), Los Angeles, CA, USA

2 Department of Radiological Sciences, David Geffen School of Medicine, University of California, Los Angeles (UCLA), Los Angeles, CA, USA

3 Department of Radiology, Keck School of Medicine, University of Sothern California (USC), Los Angeles, CA, USA

4 Department of Radiology, University Hospitals Cleveland Medical Center, Cleveland, OH, USA

5 Division of Emergency Radiology, Department of Radiology, Keck School of Medicine, University of Sothern California (USC), 1500 San Pablo Street, Los Angeles, CA 90033, USA

Steven S. Raman

Sraman@mednet.ucla.edu 
first and second quarters of 2020, with backlog recovery of many cases in the third and fourth quarters of 2020. The pandemic has unveiled and exacerbated longstanding health disparities and challenges related to healthcare access, which need to be addressed. Moreover, the COVID-19 pandemic has disrupted the ongoing cancer screening programs and has a significant downstream negative impact on patients, healthcare providers, and hospital systems. The pandemic also caused substantial challenges with trainee education, radiology research, and meetings.

The aim of this manuscript is to highlight the challenges the radiology community faced over the past year and the impact the pandemic had on radiology staff, radiology practices, trainee education, and radiology research. In addition, this manuscript aims to summarize the lessons learned from the pandemic and to prepare the radiology community for the post-COVID-19 era.

\section{Methods}

The present study was prepared based on the joint opinions of radiology experts from three major US academic institutions. For the purpose of this study, three independent researchers systematically searched PubMed (MEDLINE), Scopus, Embase (Elsevier), Web of Science, and Google Scholar databases for published original articles or systematic reviews on COVID-19 between January 1, 2020, and February 28, 2021. Keywords for the literature search included "coronavirus disease," "COVID-19," "radiology practice," "cancer screening," "radiology training," "radiology residents," "radiology meetings," "radiology research," and "well-being." Papers with unrelated content, nonavailable full texts, suspicious of duplicate or non-scientific material, and those published in other languages rather than English were excluded from the final review. Overall, more than 100 related published papers were considered and 42 related published papers were included in the final review by all authors, and then the paper was drafted and revised critically multiple times based on the opinions of the authors.

\section{COVID-19 pandemic and radiology practice}

The early weeks of the pandemic were highlighted with swift alterations in the radiology workforce, as there was uncertainty around the clinical indications and role of imaging in individuals with COVID-19. The unprecedented situation required flexibility and prioritization of the resources in radiology departments. During that time, webinar-based teachings and sharing of information through social media helped the radiology community adapt to the rapidly changing protocols [4]. Also, with radiologists transitioning to remote readouts, departments and practices had to develop or improve teleradiology infrastructure in a timely manner.

Several studies have explored the economic effects of the COVID-19-imposed restrictions, stay-at-home orders, and delayed non-urgent care on radiology practices. The number of imaging studies in both academic and private sectors was substantially decreased in the early months of the pandemic. A survey conducted among members of ACR, and Radiology Business Management [5] found a 56.4-63.7\% decline in imaging volumes during April 2020 with 50.3\% of practices reducing their staff salaries. A large healthcare system in New York found the greatest decrease in the volume of imaging studies in mammography (94\%), nuclear medicine (85\%), MRI (74\%), and ultrasound (64\%), followed by interventional radiology (56\%), CT (46\%), and x-ray (22\%) [6]. A significant increase in the number of no-show radiology visits was also reported during April 2020 compared to the similar time period in 2019 (49.9\% vs. 16.9\%) [7]. Similarly, cancer screening programs were decimated by the pandemic, resulting in an unprecedented decrease in unscreened patients. Reported decreases in rates of breast, colon, and cervical cancer screening were 94\%, 86\%, and 94\% respectively between January and April 2020 [8]. The drop in the volume of imaging studies for cancer screening might have caused missed prevention opportunities and delay in the diagnosis of cancer; however, the long-term scopes are still unknown. Innovative strategies would be required to bridge the gap of lost screening opportunity and enhance the existing screening programs, including usage of mobile mammography equipment [9], proactive outreach of the high-risk patients, use of social media $[8,10]$ to communicate risks of missed cancer to the general population, and initial in-person assessment with follow-up via telemedicine.

Despite the early unprecedented and precipitous decrease in diagnostic imaging in Q1 and Q2, 2020, the volume of imaging studies increased slowly toward baseline in Q3 2020, and by the end of September 30, 2020, CT volume was $84 \%$ of predicted, according to data from 2398 radiology practices across the USA [11]. While the risk of contracting COVID-19 for patients using ambulatory healthcare facilities is low, the incomplete recovery of imaging volume may be caused by continued concerns about safety, social distancing, or the loss of individual health insurance from unemployment.

The private practice sector, and specifically smaller groups, was severely affected by the economic strains of the pandemic. To financially survive the COVID-19 pandemic, private practices had to adjust their costs, including decreased physician compensation, paid leave, bonuses and benefits, new hiring freezes and even radiologist layoffs. To mitigate financial losses, investments in new technology and cutting-edge equipment were deferred or eliminated by many institutions [12]. Coronavirus relief funds helped 
many healthcare institutions, including radiology practices, survive the pandemic. Further details of these funds are beyond the scope of this review.

While the workflow of most of the radiology practices has returned to the pre-pandemic levels, many practices have retained the developed infrastructure for remote working. The widespread adoption of teleradiology will likely result in radiology practice restructuring that favors larger multispecialty regional and national groups. For individual radiologists, there may be a better work-life balance at the expense of increased social and professional isolation.

\section{COVID-19 pandemic effect on radiology training}

Decreased radiology resident and fellow imaging and procedure volumes, and the shift to remote workflow of attending radiologists, may cause a significant negative impact on trainee education if implemented without adequate training safeguards. In response to professional distancing recommendations and to prevent overcrowding, many institutions limited or eliminated trainee involvement in hands-on skills such as fluoroscopy, ultrasound, and image-guided procedures early in the COVID-19 pandemic. At one large academic institution early in the pandemic, the number of on-site radiology residents was decreased by $60 \%$ (from 50 to 20) during the workweek [10]. Daily readouts are an essential component of the training in radiology residency programs. Most academic radiology departments evolved from traditional side-by-side trainee-attending reading configurations to individual reading remotely in synchronous or asynchronous configurations on online platforms. While the asynchronous remote readouts give a higher degree of autonomy and confidence to the trainee and are positively received by both trainees and attendings, both parties have experienced some apprehension about loss of direct teaching, poor professional relationship, and loss of social interaction during remote readouts [13]. The use of asynchronous readouts specifically in senior trainees might continue beyond the pandemic. On a survey of 107 radiology residency programs, $70 \%$ reported a moderate or marked negative impact of the COVID-19 pandemic on their departments' educational missions [14].

Residency training programs' didactic lectures were migrated from in-person sessions rapidly to the virtual environment on online platforms. Advantages of this system include the ability to interact across sites, availability of recorded content for later study, ability to invite high caliber speakers from around the world with use of extensive educational resources which were made available for free by RSNA, ARRS, and other subspecialty societies, and flexibility for conference timing. The main disadvantage is the decreased interaction of the trainees with the lecturers on virtual platforms and the daily social and professional interaction inherent of in-person meetings. In a survey of 140 radiology chief residents [15], 63\% preferred in-person educational lectures over videoconferences.

The physical, mental, and emotional well-being of the radiology community has also been affected by the pandemic. According to a survey of radiology residency programs, fear of self or loved ones contracting SARS-CoV-2 and a feeling of being isolated were the two most common reasons for decreased resident morale in $78.5 \%$ and $74.8 \%$ of responders respectively [14]. Furthermore, morale and well-being were also significantly impacted when radiology residents were redeployed into non-radiology clinical roles. Trainees also expressed concerns about the postponed American Board of Radiology (ABR) CORE exam, potentially lost educational opportunities, and uncertainly about their future job prospects.

\section{COVID-19 pandemic effect on radiology research and scientific meetings}

During the COVID-19 pandemic, most of the radiological research studies were either halted or slowed down considerably, except those related to COVID-19 imaging. The ACR statement indicated research-oriented imaging studies should have the least priority during the early reopening of departments. Many imaging studies for research purposes have gradually but incompletely restarted; however, in areas with a rise in the number of cases, they have been temporarily stopped again. Most national and international radiology meetings and conferences were canceled or moved to virtual platforms after the declaration of the pandemic. While virtual-only conferences were a great opportunity to watch scientific and on-demand educational lectures with location and time flexibility, the nature of virtual-only meetings had a negative impact on many other aspects of the meetings, including networking opportunities, career development, vendor marketing, sharing of research work results, and further collaborations [16]. While there have been substantial improvements with virtual meeting platforms to keep the attendees engaged, the major drawback that has still remained in place is the lack of "social interaction," "catching up," and "networking" components which are an essential part of modern-day meetings. The virtual platforms have substantially improved during the past year by offering more interactive solutions. The in-person radiology meetings are restarting; however, it is likely that the developed virtual platforms remain in place for major societal meetings to offer an alternative for those who cannot attend the inperson meeting. The hybrid scientific meetings with a combined in-person and virtual platforms can offer the benefits 
of both formats. This further necessitates the development of solutions for the more interactive role of the attendees in virtual platforms.

\section{What lessons have been learned and what to expect?}

\section{Radiology practice and radiology training}

As more radiologists are adapting to remote readings, many academic and private radiology practices will likely be altered permanently. While this may lead to a better worklife balance, it may affect trainee-attending collaboration. In addition, increased isolation may augment symptoms of anxiety, depression, and burnout for some. The overall impact on radiologists' well-being will need to be further studied.

\section{Radiology scientific meetings}

Despite substantial improvements in virtual platforms, these platforms still lack the "in-person" social interactions and networking. Since the virtual components of lectures, conferences, and meetings are likely to persist, a hybrid format including both "in-site" and "virtual" attendance could be a "new normal."

\section{Preparedness for the future}

The COVID-19 pandemic is not the first pandemic and won't be the last. Healthcare systems should maintain the newly developed infrastructures and HCW should be regularly trained to stay prepared for future pandemics. In addition, hospital systems and healthcare policymakers should ensure an adequate stockpile of PPE for emergency use. The COVID-19 experience showed that radiologists need to stay alert for the new outbreaks from unknown vectors, often first detected on imaging. Radiologists interpret high volumes of patients each day and since most new contagious outbreaks cause respiratory syndromes, radiologists should be on the lookout for suspicious repetitive imaging patterns in a short period of time, which are not justified by the expected imaging patterns of known local infectious agents.

Author contribution Literature review (all authors), manuscript preparation (SK, MH, EV), manuscript critical review and revision (SR, AG, $\mathrm{RG}, \mathrm{AG}$ ), supervision (AG).

The authors declare that they had full access to all of the data in this study and the authors take complete responsibility for the integrity of the data and the accuracy of the data analysis.

\section{Declarations}

Conflict of interest The authors declare that they have no coflict of interest.

\section{References}

1. (WHO), W.H.O. Director-General's opening remarks at the media briefing on COVID-19 - March 11, 2020. 2020 March 11, 2020 [cited 2021 02/12/2021]; Available from: https://www.who.int/ director-general/speeches/detail/who-director-general-s-openingremarks-at-the-media-briefing-on-covid-19---11-march-2020

2. Kooraki S et al (2020) Coronavirus (COVID-19) outbreak: what the Department of Radiology should know. J Am Coll Radiol 17(4):447-451

3. Davenport MS et al (2020) ACR statement on safe resumption of routine radiology care during the coronavirus disease 2019 (COVID-19) pandemic. J Am Coll Radiol 17(7):839-844

4. Hudson BJ et al (2020) Lasting lessons learnt in the radiology department from the battle with COVID-19. Clin Radiol 75(8):586-591

5. Malhotra A et al (2020) Initial impact of COVID-19 on radiology practices: an ACR/RBMA survey. J Am Coll Radiol 17(11):1525-1531

6. Naidich JJ et al (2020) Impact of the coronavirus disease 2019 (COVID-19) pandemic on imaging case volumes. J Am Coll Radiol 17(7):865-872

7. Doshi AH et al (2021) Impact of COVID-19 social distancing regulations on outpatient diagnostic imaging volumes and noshow rates. Clin Imaging 76:65-69

8. Cancino RS, et al. (2020) The impact of COVID-19 on cancer screening: challenges and opportunities. JMIR Cancer 6(2): e21697

9. Tsai HY et al (2020) Effects of the COVID-19 pandemic on breast cancer screening in Taiwan. Breast 54:52-55

10. Plackett R, et al. (2020) Use of social media to promote cancer screening and early diagnosis: scoping review. J Med Internet Res 22(11): e21582

11. Davenport MS, et al. (2020) CT volumes from 2,398 radiology practices in the United States: a real-time indicator of the effect of COVID-19 on routine care, January to September 2020. J Am Coll Radiol

12. Sharpe RE et al (2021) Special report of the RSNA COVID19 Task Force: the short- and long-term financial impact of the COVID-19 pandemic on private radiology practices. Radiology 298(1):E11-E18

13. Matalon SA et al (2020) Trainee and attending perspectives on remote radiology readouts in the era of the COVID-19 pandemic. Acad Radiol 27(8):1147-1153

14. Robbins JB et al (2020) COVID-19 impact on well-being and education in radiology residencies: a survey of the association of program directors in radiology. Acad Radiol 27(8):1162-1172

15. Hoegger MJ et al (2021) A snapshot of radiology training during the early COVID-19 pandemic. Curr Probl Diagn Radiol 50(5):607-613

16. Evens RG (2020) The impact of a pandemic on professional meetings. Radiology: Imaging Cancer 2(3): e204012

Publisher's Note Springer Nature remains neutral with regard to jurisdictional claims in published maps and institutional affiliations. 